\title{
Permutation-based methods for mediation analysis in studies with small sample sizes
}

\author{
Miranda E Kroehl ${ }^{\text {Corresp., }}{ }^{1}$, Sharon M Lutz ${ }^{2}$, Brandie D Wagner ${ }^{1}$ \\ ${ }^{1}$ Department of Biostatistics and Informatics, Colorado School of Public Health, University of Colorado Anschutz Medical Campus, Aurora, CO, United \\ States \\ Department of Population Medicine, Harvard Medical School and Harvard Pilgrim Health Care, Harvard University, Boston, MA, United States \\ Corresponding Author: Miranda E Kroehl \\ Email address: miranda.kroehl@cuanschutz.edu
}

Background. Mediation analysis can be used to evaluate the effect of an exposure on an outcome acting through an intermediate variable or mediator. For studies with small sample sizes, permutation testing may be useful in evaluating the indirect effect (i.e. the effect of exposure on the outcome through the mediator) while maintaining the appropriate type I error rate. For mediation analysis in studies with small sample sizes, existing permutation testing methods permute the residuals under the full or alternative model, but have not been evaluated under situations where covariates are included. In this paper, we consider and evaluate two additional permutation approaches for testing the indirect effect in mediation analysis based on permutating the residuals under the reduced or null model which allows for the inclusion of covariates.

Methods. Simulation studies were used to empirically evaluate the behavior of these two additional approaches: (1) the permutation test of the Indirect Effect under R educed Models (IERM) and (2) the Permutation Supremum test under R educed M odels (PSRM). The performance of these methods was compared to the standard permutation approach for mediation analysis, the permutation test of the Indirect Effect under Full Models (IEFM). We evaluated the type 1 error rates and power of these methods in the presence of covariates since mediation analysis assumes no unmeasured confounders of the exposure-mediator-outcome relationships.

Results. The proposed PSRM approach maintained type I error rates below nominal levels under all conditions, while the proposed IERM approach exhibited grossly inflated type I rates in many conditions and the standard IEFM exhibited inflated type I error rates under a small number of conditions. Power did not differ substantially between the proposed PSRM approach and the standard IEFM approach.

Conclusions. The proposed PSRM approach is recommended over the existing IEFM approach for mediation analysis in studies with small sample sizes. 


\section{Permutation-Based Methods for Mediation Analysis in}

\section{Studies with Small Sample Sizes}

3

4 Miranda E. Kroehl ${ }^{1}$, Sharon M. Lutz ${ }^{2}$, Brandie D. Wagner ${ }^{1}$

5

$6 \quad{ }^{1}$ Department of Biostatistics and Informatics, Colorado School of Public Health, University of

7 Colorado Anschutz Medical Campus, Aurora, CO, United States

$8 \quad{ }^{2}$ Department of Population Medicine, Harvard Medical School and Harvard Pilgrim Health

9 Care, Harvard University, Boston, MA, United States

10

11 Corresponding Author:

12 Miranda E. Kroehl ${ }^{1}$

1313001 East $17^{\text {th }}$ Place, B119-406, Aurora, CO 80045

14 Email address: miranda.kroehl@ucdenver.edu 


\section{ABSTRACT}

16 Background. Mediation analysis can be used to evaluate the effect of an exposure on an

17 outcome acting through an intermediate variable or mediator. For studies with small sample

18 sizes, permutation testing may be useful in evaluating the indirect effect (i.e. the effect of

19 exposure on the outcome through the mediator) while maintaining the appropriate type I error

20 rate. For mediation analysis in studies with small sample sizes, existing permutation testing

21 methods permute the residuals under the full or alternative model, but have not been evaluated

22 under situations where covariates are included. In this paper, we consider and evaluate two

23 additional permutation approaches for testing the indirect effect in mediation analysis based on

24 permutating the residuals under the reduced or null model which allows for the inclusion of

25 covariates.

26 Methods. Simulation studies were used to empirically evaluate the behavior of these two

27 additional approaches: (1) the permutation test of the Indirect Effect under Reduced Models

28 (IERM) and (2) the Permutation Supremum test under Reduced Models (PSRM). The

29 performance of these methods was compared to the standard permutation approach for

30 mediation analysis, the permutation test of the Indirect Effect under Full Models (IEFM). We

31 evaluated the type 1 error rates and power of these methods in the presence of covariates

32 since mediation analysis assumes no unmeasured confounders of the exposure-mediator-

33 outcome relationships.

34 Results. The proposed PSRM approach maintained type I error rates below nominal levels

35 under all conditions, while the proposed IERM approach exhibited grossly inflated type I rates in

36 many conditions and the standard IEFM exhibited inflated type I error rates under a small

37 number of conditions. Power did not differ substantially between the proposed PSRM approach

38 and the standard IEFM approach. 
39 Conclusions. The proposed PSRM approach is recommended over the existing IEFM approach

40 for mediation analysis in studies with small sample sizes.

41 Keywords: mediation analysis, product of coefficients test, intersection union test, permutation

42 under full model, permutation under reduced model 


\section{INTRODUCTION}

Mediation analysis can be used to evaluate whether the exposure acts on the outcome

through an intermediate variable (i.e. the mediator). The product of two regression coefficients can be used to estimate and test this indirect effect (Baron \& Kenny, 1986). However, because the product of two normally distributed random variables, such as two regression coefficients, is not normally distributed and does not approximate commonly used distributions, testing the indirect effect requires careful consideration (Aroian, 1944).

There have been a variety of tests proposed to evaluate the indirect effect in mediation analysis, including parametric methods (MacKinnon, Lockwood, Hoffman, West, \& Sheets, 2002; MacKinnon, Lockwood, \& Williams, 2004; Williams \& MacKinnon, 2008; Hayes, 2009; Taylor \& MacKinnon, 2012; Koopman, Howe, Hollenbeck, \& Sin, 2015) and resampling methods (VanderWeele, 2014; MacKinnon et al., 2002; MacKinnon et al., 2004; Williams \& MacKinnon, 2008; Hayes, 2009; Taylor \& MacKinnon, 2012; Koopman, et al., 2015). However, for studies with small sample sizes, bootstrapping approaches can result in inflated Type I error rates and permutation testing has been proposed as an alternative resampling approach (Koopman et al., 2015; Tofighi \& MacKinnon, 2011; Williams \& MacKinnon, 2008).

Special consideration is needed for permutation testing in mediation analysis. Due to the assumption of no unmeasured confounding in mediation analysis, covariates need to be included to account for any confounding of the exposure-mediator-outcome relationships. The inclusion of covariates can add complexity to permutation approaches and the standard approach of permuting raw values is not appropriate for this situation. Permuting the outcome not only breaks the association between the exposure and outcome, but additionally breaks up the association between the outcome and any confounders, yielding a global test of all regression coefficients rather than the coefficient of interest (Anderson \& Legendre, 1999; 
67 Freedman \& Lane, 1983). Similarly, permuting the exposure will not only break the association

68 between the exposure and outcome, but would also break the associations between the

69 exposure and any confounders. An alternative to permuting the outcome or the exposure is to use the residuals from a linear model as the permutable units for the test (Anderson \&

71 Legendre, 1999). Two major approaches in the field are to permute the residuals under the 72 reduced, or null, model (Freedman \& Lane, 1983) which excludes the covariate of interest, or 73 permute the residuals under the full, or alternative, model which includes the covariate of interest (Manly, 1997; ter Braak, 1992). For example, consider a test on the regression coefficient $\beta_{1}$, where $\beta_{1}$ measures the linear association between covariate $C_{1}$ and outcome $\mathrm{Y}$ while accounting for covariate $C_{2}$, and the hull hypothesis is $H_{0}: \beta_{1}=0$. The permutation of residuals under the null model approach would permute residuals from the "null" model, $Y=\beta_{0}$ $+\beta_{2} C_{2}$, where $\beta_{1}$ is assumed to be 0 . The permutation of residuals under the full model approach would permute residuals from the "full" model $Y=\beta_{0}+\beta_{1} C_{1}+\beta_{2} C_{2}$, under the alternative assumption that $\beta_{1} \neq 0$. sample sizes permute the residuals under the full model or the raw data (Koopman et al., 2015; Taylor and MacKinnon, 2012). In order to accommodate covariates, we propose two additional permutation approaches for testing the indirect effect in mediation analysis based on permutation of residuals under the reduced model: (1) the permutation test of the Indirect Models (PSRM). Through simulation studies, we compare these 2 proposed approaches to the standard permutation approach for mediation analysis in studies with small sample sizes, the permutation test of the Indirect Effect under Full Models (IEFM) (Koopman et al., 2015; Taylor 
91 mediation analysis assumes no unmeasured confounders of the exposure-mediator-outcome

92 relationship. We illustrate the potential consequences of permuting without careful

93 consideration of the underlying assumptions and the null hypothesis, and provide

94 recommendations on using permutation testing for mediation analysis in studies with small

95 sample sizes.

96

97 METHODS

Regression-based approaches for evaluating mediation were first popularized by Baron and Kenny (1986), and extensions are now widely used in psychology and epidemiology research (Mackinnon \& Fairchild, 2009; VanderWeele, T, 2009). Let X be the exposure or

101 intervention variable, $\mathrm{M}$ the mediating or intermediate variable, $\mathrm{Y}$ the outcome of interest, and

102 C a set of covariates. The relationships between these variables are illustrated in Figure 1 and

103 defined in the following equations for the outcome $Y$ (equation 1 ) and the mediator $M$ (equation 104 2).

$$
E[Y \mid X=x, \boldsymbol{C}=\boldsymbol{c}, M=m,]=\gamma_{0}+\gamma_{1} x+\gamma_{2} \boldsymbol{c}+\gamma_{3} m
$$

$$
E[M \mid X=x, \boldsymbol{C}=\boldsymbol{c}]=\alpha_{0}+\alpha_{1} x+\alpha_{2} \boldsymbol{c}
$$

where the indirect effect (IE) can be calculated such that

$$
\widehat{I E}=\hat{\alpha}_{1} * \hat{\gamma}_{3}
$$

We propose two permutation approaches to test the indirect effect, which permutes the

111 residuals under the reduced model (Freedman \& Lane, 1983) and have not been previously

112 implemented for mediation analysis. We compare these two proposed methods to the standard

113 method for permutation testing in mediation analysis for studies with small sample sizes, which

114 permutes the residuals under the full model (Koopman et al., 2015; Taylor and MacKinnon, 
115 2012). The methods for all 3 approaches can be used for both exact and approximate

116 permutation tests, depending on the number of permutations specified $\left(n_{\text {perm }}\right)$. An exact

117 permutation test calculates a test statistic for all possible permuted samples, whereas an

118 approximate permutation test calculates a subset of samples.

Proposed Approach 1: Permutation test of the Indirect Effect under Reduced Models

121 (IERM)

122 The permutation test of the indirect effect under reduced models (IERM) makes use of the

123 Freedman and Lane approach for permuting the residuals (Freedman \& Lane, 1983) from the

124 reduced model. Using their general framework, the IERM approach fits full models for

125 Equations 1 and 2 in order to obtain an estimate for the indirect effect (equation 3). Two

126 reduced models are fit excluding the parameters used to calculate the indirect effect and

127 residuals from each are calculated and permuted. This approach is intended to break the

128 associations between $\mathrm{M}$ and $\mathrm{Y}$, and $\mathrm{X}$ and $\mathrm{M}$, without disturbing associations with $\mathrm{C}$, thus

129 creating an estimate of the null distributions for $\alpha_{1}$ and $\gamma_{3}$, and of $\alpha_{1} * \gamma_{3}$.

130 The proposed IERM approach implements the following steps:

131 1. Fit the full models: $\quad Y=\hat{\gamma}_{0}+\hat{\gamma}_{1} X+\hat{\gamma}_{2} C+\hat{\gamma}_{3} M+e_{Y}$

1332 2. Estimate the original estimate for the indirect effect, $\left(\hat{\alpha}_{1} * \hat{\gamma}_{3}\right)_{\text {orig }}$

134 3. Fit reduced models: $\quad Y=\hat{\gamma}_{0(r)}+\hat{\gamma}_{1(r)} X+\hat{\gamma}_{2(r)} C+e_{Y(r)}$

$$
M=\hat{\alpha}_{0(r)}+\hat{\alpha}_{2(r)} C+e_{M(r)}
$$

4. Using the reduced models from Step 3, estimate $\hat{Y}, e_{Y(r)}, \hat{M}$, and $e_{M(r)}$

5. Permute residuals from the reduced models, now labeled $e_{Y}{ }^{*}$ and $e_{M}{ }^{*}, \mathrm{n}_{\text {perm }}$ times and for each permutation, calculate $Y^{*}=\hat{Y}+e_{Y}{ }^{*}$ and $M^{*}=\hat{M}+e_{M}{ }^{*}$. 
6. For each permutation, fit the regression models from step 1, replacing $Y$ and $M$ with $Y^{*}$ and $M^{*}$ respectively. Calculate $\hat{\alpha}_{1}{ }^{*} * \hat{\gamma}_{3}{ }^{*}$.

7. The absolute value of $\left(\hat{\alpha}_{1} * \hat{\gamma}_{3}\right)_{\text {orig }}$ is compared to the distribution of absolute values of $\hat{\alpha}_{1}{ }^{*} * \hat{\gamma}_{3}{ }^{*}$ (for a two-tailed test). The $\mathrm{p}$-value is calculated as the proportion of values in the distribution that have equal or greater magnitudes than $\left(\hat{\alpha}_{1} * \hat{\gamma}_{3}\right)_{\text {orig }}$, i.e. whose absolute values are greater than or equal to the absolute value of $\left(\hat{\alpha}_{1} * \hat{\gamma}_{3}\right)_{\text {orig }}$.

\section{Proposed Approach 2: Permutation Supremum test under Reduced Models (PSRM)}

The permutation supremum test under reduced models (PSRM) also makes use of the

148 Freedman and Lane permutation approach (Freedman \& Lane, 1983), and incorporates a supremum testing approach for mediation by Wagner et al. (2017) to evaluate the composite null hypothesis $\alpha_{1} * \gamma_{3}=0, \alpha_{1}=0$ and $\gamma_{3}=0$. The PSRM differs from the IERM above in that

151 the individual coefficients along the proposed pathway, $\hat{\alpha}_{1}$ and $\hat{\gamma}_{3}$, are tested along with the

152 indirect effect. In order to conclude that mediation is present, $\hat{\alpha}_{1} * \hat{\gamma}_{3}, \hat{\alpha}_{1}$ and $\hat{\gamma}_{3}$ must be

153 significantly different from zero.

154 The proposed PSRM differs from the IERM in Steps 2, 6, and 7, and implements the 155 following steps:

156 1. Fit the full models: $\quad Y=\hat{\gamma}_{0}+\hat{\gamma}_{1} X+\hat{\gamma}_{2} C+\hat{\gamma}_{3} M+e_{Y}$

$$
M=\hat{\alpha}_{0}+\hat{\alpha}_{1} X+\hat{\alpha}_{2} C+e_{M}
$$

2. Estimate the original estimates $\hat{\alpha}_{1 \text { orig }}, \hat{\gamma}_{3 \text { orig }}$ and $\left(\hat{\alpha}_{1} * \hat{\gamma}_{3}\right)_{\text {orig }}$.

3. Fit reduced models: $\quad Y=\hat{\gamma}_{0(r)}+\hat{\gamma}_{1(r)} X+\hat{\gamma}_{2(r)} C+e_{Y(r)}$

$$
M=\hat{\alpha}_{0(r)}+\hat{\alpha}_{2(r)} C+e_{M(r)}
$$

4. Using the reduced models from Step 3, estimate $\hat{Y}, e_{Y(r)}, \hat{M}$, and $e_{M(r)}$ 
162

164

166

175

\section{6}

177

178

179

180

181

182

183 184

5. Permute residuals from the reduced models, now labeled $e_{Y}{ }^{*}$ and $e_{M}{ }^{*}, \mathrm{n}_{\text {perm }}$ times and for each permutation, calculate $Y^{*}=\hat{Y}+e_{Y}{ }^{*}$ and $M^{*}=\hat{M}+e_{M}{ }^{*}$.

6. For each permutation, fit the regression models from step 1, replacing $Y$ and $M$ with $Y^{*}$ and $M^{*}$ respectively. Estimate $\hat{\alpha}_{1}{ }^{*} * \hat{\gamma}_{3}{ }^{*}, \hat{\alpha}_{1}{ }^{*}$ and $\hat{\gamma}_{3}{ }^{*}$.

7. The absolute value of $\left(\hat{\alpha}_{1} * \hat{\gamma}_{3}\right)_{\text {orig }}$ is compared to the distribution of absolute values of $\hat{\alpha}_{1}{ }^{*} * \hat{\gamma}_{3}{ }^{*}$ (for a two-tailed test). The $\mathrm{p}$-value is calculated as the proportion of values in the distribution that have equal or greater magnitudes than $\left(\hat{\alpha}_{1} * \hat{\gamma}_{3}\right)_{\text {orig }}$. The absolute value of $\hat{\alpha}_{1 \text { orig }}$ is compared to the distribution of absolute values of $\hat{\alpha}_{1}{ }^{*}$ (for a two-tailed test). The $\mathrm{p}$-value is calculated as the proportion of values in the distribution that have equal or greater magnitudes than $\hat{\alpha}_{1 \text { orig. }}$. Similarly, a $\mathrm{p}$-value is obtained for $\hat{\gamma}_{3 \text { orig. }}$. The null hypothesis is rejected only if $\left(\hat{\alpha}_{1} * \hat{\gamma}_{3}\right)_{\text {orig }}, \hat{\alpha}_{1 \text { orig }}$ and $\hat{\gamma}_{3 \text { orig }}$ are significantly different from zero, and the significance level is the supremum of the significance levels of the individual tests.

\section{Approach for Comparison: The permutation test of the Indirect Effect under Full}

\section{Models (IEFM)}

The permutation test for the indirect effect under the full models (IEFM) makes use of ter Braak's method (Manly, 1997; ter Braak, 1992), fitting full models for both regression models and estimating the indirect effect. Rather than estimating both full and reduced models as in the PSRM and IEFM, only the full models are fitted in this approach. Residuals are permuted and used to create a sampling distribution of the test statistic and estimate confidence limits for the indirect effect (Koopman et al., 2015; Taylor and MacKinnon, 2012).

The IEFM method implements the following steps: 
185

186

188

196

197

200

201

202

203

204

1. Fit the full models: $\quad Y=\hat{\gamma}_{0}+\hat{\gamma}_{1} X+\hat{\gamma}_{2} C+\hat{\gamma}_{3} M+e_{Y}$

$$
M=\hat{\alpha}_{0}+\hat{\alpha}_{1} X+\hat{\alpha}_{2} C+e_{M}
$$

2. Estimate the original estimate for the indirect effect, $\left(\hat{\alpha}_{1} * \hat{\gamma}_{3}\right)_{\text {orig }}$

3. Permute residuals from the full models, now labeled $e_{Y}{ }^{*}$ and $e_{M}{ }^{*}, \mathrm{n}_{\text {perm }}$ times and for each permutation, calculate $Y^{*}=\hat{Y}+e_{Y}{ }^{*}$ and $M^{*}=\hat{M}+e_{M}{ }^{*}$.

4. For each permutation, fit the regression models from step 1, replacing $Y$ and $M$ with $Y^{*}$ and $M^{*}$ respectively. Calculate $\hat{\alpha}_{1}{ }^{*} * \hat{\gamma}_{3}{ }^{*}$.

5. Confidence bounds are estimated using the $\left(\frac{\omega}{2}\right) * 100$ and $\left(1-\frac{\omega}{2}\right) * 100$ percentiles of the distribution of $\hat{\alpha}_{1}{ }^{*} * \hat{\gamma}_{3}{ }^{*}$, where $\omega$ corresponds to the desired alpha level.

6. The null hypothesis, $\left(\hat{\alpha}_{1} * \hat{\gamma}_{3}\right)_{\text {orig }}=0$, is rejected if 0 is not contained within the confidence bounds.

\section{SIMULATION STUDIES}

Simulation studies were used to empirically evaluate the behavior of the two proposed approaches (IERM and PSRM) and the existing IEFM method (Koopman et al., 2015; Taylor and MacKinnon, 2012). To ensure scenarios of weak and strong confounding, normally distributed data were generated for $\mathrm{X}, \mathrm{M}, \mathrm{Y}$, and $\mathrm{C}$ from a range of correlation structures by multiplying the Cholesky decomposed matrix of the correlation structure with a matrix of independent and random normally distributed values. A subset of all possible correlations of $0,0.15,0.3$, and 0.6 between the four variables with positive definite correlation structures was evaluated (see online supplement Tables S1 and S2 for complete list of scenarios and the corresponding regression coefficients). Correlation strengths were selected to simulate comparable regression coefficients to prior studies on permutation methods for mediation (Taylor \& MacKinnon, 2012); 
208 the simulated regression coefficients are reported in the online supplement. For example, one

209 condition evaluated was a scenario where $C$ was a weak confounder between $X$ and $Y$, with a

210 correlation structure of $\begin{array}{r}X \\ C\end{array}\left[\begin{array}{cccc}1 & 0.15 & 0.15 & 0.15 \\ \cdots & 1 & 0 & 0.15 \\ Y & \ldots & 1 & 0.15 \\ \cdots & \cdots & \ldots & 1\end{array}\right]$

211 Three covariate or confounding scenarios were considered in this study: 1) C as a

212 covariate, associated with outcome $Y$ but not with the exposure $X$ or mediator $M$ (Figure 2a), 2)

213 C as a confounder of the exposure-outcome ( $\mathrm{X}-\mathrm{Y}$ ) relationship (Figure $2 \mathrm{~b}$ ), and 3) C as a

214 confounder of the mediator-outcome (M-Y) relationship (Figure $2 \mathrm{c}$ ). For each scenario, $\mathrm{C}$ was

215 assessed as either a "weak" or "strong" covariate or confounder, with correlation of 0.15 or 0.6

216 respectively, resulting in a total of 6 covariate scenarios evaluated.

217 Under the null of no indirect effect, type I error rates were evaluated at a significance

218 level of $a=0.05$. The null hypothesis of no indirect effect may be simulated from the following

219 three scenarios: (1) both $\alpha_{1}=0$ and $\gamma_{3}=0,(2) \alpha_{1}=0$ and $\gamma_{3} \neq 0$, or (3) $\alpha_{1} \neq 0$ and $\gamma_{3}=0$. All

220 three null possibilities were evaluated for $\mathrm{C}$ as a weak or strong covariate or confounder. All

221 other correlations were held at 0.15 for the "weak" scenario, and 0.6 for the "strong" scenario,

222 resulting in a total of 27 conditions (13 conditions for scenario a, 8 conditions for scenario b,

223 and 6 conditions for scenario c) per sample size being evaluated under the null.

224 Under the alternative hypothesis, both $\alpha_{1} \neq 0$ and $\gamma_{3} \neq 0$. Correlation between the exposure $\mathrm{X}$ and the mediator $\mathrm{M}, \rho_{X M}$, or correlation between the mediator $\mathrm{M}$ and outcome $\mathrm{Y}$, $\rho_{M Y}$, varied from $0.15,0.3$, and 0.6 . For each of the 6 covariate scenarios, $\rho_{X M}$ and $\rho_{M Y}$ were varied as $0.15,0.3$, or 0.6 , while all other correlations were held at 0.15 for the "weak" scenario, and 0.6 for the "strong" scenario, resulting in a total of 51 conditions (18 conditions

229 for scenario a, 18 conditions for scenario b, and 15 conditions for scenario c) per sample size 230 being evaluated under the alternative. Similar to Koopman et al. (2015), we considered sample 
231 sizes of 30 and 100 . For each simulation condition, 1,000 replicates were run with 10,000

232 permutations per replicate. Simulation code is available at

233 https://github.com/kroehlm/Permutation_Mediation_Test.

234

235 RESULTS

236

Across the three covariate scenarios, results were very similar for conditions in which the

coefficients $\alpha_{1}$ and $\gamma_{3}$ were mirrored; i.e. the results when $\alpha_{1}=0.15$ and $\gamma_{3}=0$ were similar to

238 those for $\alpha_{1}=0$ and $\gamma_{3}=0.15$. For simplicity, only the results for one set of covariate

239 scenarios, $\mathrm{C}$ as a confounder of the exposure-outcome (X-Y) relationship, will be presented;

240 results from all scenarios are in the online supplement.

241 Type I error

Type I error rates with $\mathrm{C}$ as a confounder between the exposure-outcome $(\mathrm{X}-\mathrm{Y})$

243 relationship are shown in Table 1. Results are shown in Table 1 for the null scenario (1) in

244 which no relationship exists between the exposure-mediator (X-M) and mediator-outcome (M-Y)

245 (i.e. $\alpha_{1}=0, \gamma_{3}=0$ ) and the null scenario (2) where no relationship exists between the

246 exposure-mediator (X-M) (i.e. $\alpha_{1}=0$ and $\gamma_{3} \neq 0$ ). The null scenario (3) where no relationship

247 exists between mediator-outcome (M-Y) (i.e. $\alpha_{1} \neq 0$ and $\gamma_{3}=0$ ) are available in the online

248 supplement. Each row of Table 1 represents a single simulation condition, and for each

249 condition, the table includes the correlation conditions among all variables, the corresponding

$250 \alpha_{1}$ and $\gamma_{3}$ coefficient values, and the empirical type I error rates for the three different

251 permutation applications.

252 Type I rates were generally slightly higher when C was a strong confounder compared

253 to when it was a weak confounder. The results in the first row of Table 1 represent the scenario

254 where $\mathrm{C}$ is a weak confounder between the $\mathrm{X}-\mathrm{Y}$ relationship, the type I error rate for the test of 
255 the indirect effect under the reduced models, the IERM approach, was 0.044 . The proposed

256 PSRM approach and the existing IEFM approach had more conservative type I error rates of

2570.005 and 0.006 , respectively. Rates for all three methods increased for conditions with a 258 strong confounder.

259 The IERM approach had type I rates at the nominal level when both $\alpha_{1}$ and $\gamma_{3}$ were

260

261

262

263

264

265

266

267

268

269

270

271

272

273

274

275

276

zero. When either $\alpha_{1}$ or $\gamma_{3}$ was nonzero, the type I error rates exceeded the nominal levels, with rates increasing with increasing size of the nonzero coefficient, and reaching levels as high as 0.653 for a sample size of 30 and 0.783 for a sample size of 100 . Both the proposed PSRM approach and the existing IEFM approach had type I error rates well below the nominal level when both $\alpha_{1}$ and $\gamma_{3}$ were zero. When either $\alpha_{1}$ or $\gamma_{3}$ was nonzero, the type I error rates increased with increasing size of the nonzero coefficient, and was generally around the nominal level when the nonzero coefficient was large. However, when the sample size was small (i.e. 30), nominal levels were exceeded a handful of times for the IEFM approach and only once for the proposed PSRM approach. For a larger sample size, type I error rates remained below any expected deviations from nominal levels with one exception; the error rates were slightly inflated for the scenario where $\mathrm{C}$ was a weak confounder between $\mathrm{X}$ and $\mathrm{Y}$, and $\gamma_{3}$ was large for the IEFM test (condition 12 in Table 1) but not for the proposed PSRM approach. Across the 54 null conditions evaluated, type I error rates were exceeded in $3.7 \%$ (2) of the conditions for the PSRM, and in $14.8 \%$ (8) of the conditions for the IEFM. With a smaller sample size, the IEFM approach occasionally had slightly higher rates than those for the proposed PSRM approach. However, for the larger size of $n=100$, the error rates between the two tests were very similar, with neither consistently being larger than the other. 


\section{Power}

278 Results for power when $C$ was a confounder between the $X-Y$ relationship are shown in

279 Table 2 (similar results for the other covariate scenarios are in Tables S5 and S6 of the online

280 supplement). Due to the highly inflated error rates for the proposed IERM approach, we

281 excluded results of power from this approach. As with the type I error rates, there were no

282 major differences of power based on the covariate scenario within a permutation method. As

283 with table 1 , each row represents a single simulation condition, and for each condition, the

284 table includes the correlations among all variables, the corresponding $\alpha_{1}$ and $\gamma_{3}$, and the power

285 for the two permutation approaches. The results in the first row represent the scenario where

286 C is a weak confounder between the $X-Y$ relationship, under the alternative condition that both

$287 \alpha_{1}$ and $\gamma_{3}$ are nonzero. As a weak confounder between $\mathrm{X}-\mathrm{Y}$, the correlations between $\mathrm{X}-\mathrm{C}$ and

$288 \mathrm{C}-\mathrm{Y}$ were simulated to be 0.15 , with zero correlation between C-M. The correlation between $\mathrm{X}-$

$289 \mathrm{Y}$ was also simulated to be 0.15 . The correlations between $\mathrm{X}-\mathrm{M}$ and $\mathrm{M}-\mathrm{Y}$ were both simulated

290 to be 0.15 , with corresponding coefficients of $\alpha_{1}=0.1535$ and $\gamma_{3}=0.1335$. For this condition,

291 the power for the proposed PSRM approach was 0.014, and power for IEFM approach was

2920.019.

293 As expected, power was lowest for small values of a coefficient, and increased with

294 increasing values of the coefficients. For the small sample size (i.e. $n=30$ ), power was typically

295 larger for the IEFM approach, especially for larger coefficient values. In the 51 alternative

296 conditions evaluated for this sample size, power for the PSRM was only equal to the IEFM in

297 one condition, and was, on average, $2.2 \%$ lower than power for the IEFM. Once the sample

298 size was increased to 100, however, there were no differences in power between the two tests;

299 see Figure 3. Power for the PSRM was equal or better than the IEFM in 25 of the 51 conditions

300 for this sample size, with a mean difference between the two methods of $0.31 \%$. Figure 4 
301 displays the change in power of the proposed PSRM approach for both weak and strong

302 confounders, based on their coefficient values, for $\mathrm{n}=100$ where $\mathrm{C}$ was a confounder between

303 the $X-Y$ relationship.

304 Comparison to Bootstrap Based Approaches for Mediation Analysis

305 Prior evaluations of the type I error rates for the IEFM approach were not inflated as

306 indicated by simulation studies (Koopman et al., 2015; Taylor \& MacKinnon, 2012). Upon

307 completion of our studies and finding the IEFM and PSRM did exceed nominal rates under a

308 small set of conditions, we conducted a post-hoc study to compare these methods with

309 bootstrap methods under the four most extreme null conditions we observed, as well as six

310 alternative conditions. The purpose of these studies was to provide a side-by-side comparison

311 of the methods reported by Koopman et al. (2015) and the permutation methods evaluated

312 here under identical conditions with weak and strong confounding. Simulations were carried

313 out as described above, and the indirect effect was evaluated by the PSRM, IEFM, and the three

314 bootstrap methods evaluated in Koopman et al. (2015): the percentile bootstrap (PB), the bias

315 corrected bootstrap (BCB), and the bias corrected accelerated bootstrap (BCAB). With respect

316 to type I error, results from these studies (Table 3) indicate the PB approach performs best

317 among the bootstrap methods, while the $B C B$ and $B C A B$ approaches exceeded nominal levels

318 under all 4 scenarios. Overall, the PSRM approach performed better than the IEFM and PB

319 approaches, maintaining error rates at the nominal level in all but one condition. Comparisons

320 of power among the five methods are presented in Table 4. For all conditions evaluated, power

321 was highest for the $B C B$ and $B C A B$, the two methods which exhibited inflated type I errors

322 under all four null conditions. Power was slightly higher for the IEFM when compared with the

323 PSRM and PB when $\mathrm{n}=30$, and comparable among the three methods when $\mathrm{n}=100$. 
324 Example: How Framing of Media Stories Influences Attitudes Regarding

325 Immigration Policy

326 We now illustrate the application of the PSRM and IEFM with an empirical example

327 based on the framing data of Brader, Valentino, \& Suhay (2008). In this study, participants

328 were randomly assigned to different media stories about immigration with either positive or

329 negative framing and asked about their attitudes and political behavior with respect to

330 immigration policy. They hypothesized that anxiety mediates the relationship between framing

331 and whether a participant would agree to send a letter about immigration policy to his or her

332 member of Congress. Their analysis, controlling for education, age, income, and sex,

333 suggested anxiety does act as a significant mediator between the two variables. Here, we will

334 extend the primary analysis by considering the role of framing on attitudes toward increased

335 immigration, a four-point item with larger vales indicating more negative attitudes. Despite

336 randomization, there was some unevenness of distribution across education groups with respect

337 to treatment exposure, so we stratify analyses by education level. Similar to Brader et al., we

338 adjusted for age, income and sex.

339 Results from our extended analysis suggest that anxiety may mediate the relationship

340 between framing and attitudes toward immigration in some, but not all, education groups. For

341 subjects with a high school education, both the p-value from the PSRM and 95\% CI from the

342 IEFM were close to their respective decision points, but did not exceed thresholds to reject the

343 null hypothesis (Table 5). For all education levels, inference between the two permutation

344 methods did not differ.

345

346 DISCUSSION

We have proposed two alternative methods for testing the presence of an indirect effect 
348 using permutation approaches under the reduced model (IERM and PSRM), and compared

349 these approaches to an existing permutation approach for mediation analysis under the full

350 model (IEFM) (Koopman et al., 2015; Taylor \& MacKinnon, 2012). An important assumption in

351 mediation analysis is that of no unmeasured confounders, the two methods we evaluated were

352 based on Freedman and Lane's permutation of residuals in order to appropriately accommodate

353 covariate adjustment. These methods have not previously been evaluated. Furthermore, our

354 simulation studies considered different covariate and confounding scenarios. We evaluated the

355 different approaches to testing the indirect effect in mediation analysis with small sample sizes.

356 While the PSRM approach has similar power to the existing IEFM approach, the proposed PSRM

357 approach had a lower type I error rate than both the existing IEFM approach and the proposed

358 IERM approach, especially for a small sample size of 30.

359 In contrast to the proposed IEFM approach, the proposed PSRM approach has the

360 advantage of being able to directly evaluate all three components to the complex, composite

361 null hypothesis that one or both coefficients along the mediated path are zero in order to test

362 the presence of the indirect effect. While the proposed PSRM approach has similar or slightly

363 less power than the existing IEFM approach (Koopman et al., 2015) for a sample size of 30, this

364 was not unexpected considering the type I errors were also higher in the IEFM approach.

365 Furthermore, for a sample size of 100 , no major differences in power were noted for the

366 proposed PSRM and the existing IEFM approaches. Further, when compared to bootstrap

367 methods, the PSRM outperformed all three methods with respect to maintaining nominal type I

368 error rates, and no major differences in power were found between the PSRM and the PB,

369 which was the only bootstrap method that did not exceed nominal type I error rates under all

370 conditions evaluated.

371 The proposed IERM approach is not recommended due to the inflated type I error rate. 
372 The poor performance of the IERM approach is due to discrepancy between the composite

373 hypothesis of the indirect effect and the null hypothesis under which permutation was

374 performed. The null hypothesis for the evaluation of the indirect effect is written as: $H o: \alpha_{1} * \gamma_{3}$

$375=0$. However, by using the permutation under reduced models approach, the $\mathrm{X}-\mathrm{M}$ and $\mathrm{M}-\mathrm{Y}$

376 associations were broken up in their respective regression models, and permutation was

377 performed under the null hypothesis that $\alpha_{1}=0$ and $\gamma_{3}=0$. Thus, the $\mathrm{p}$-value is the probability

378 of exceeding the observed value given that $\alpha_{1}=\gamma_{3}=0$, not $\alpha_{1} * \gamma_{3}=0$. Therefore, while the

379 test performed well under the null hypothesis of $\alpha_{1}=0$ and $\gamma_{3}=0$, when either $\alpha_{1}=0$ and $\gamma_{3}$

$380 \neq 0$, or $\alpha_{1} \neq 0$ and $\gamma_{3}=0$ was true, false significance for the indirect effect was achieved far too

381 often. As noted by others, it is imperative to carefully consider and permute under the correct

382 null hypothesis (Anderson \& Legendre, 1999; Churchill \& Doerge, 2008; Westfall \& Young,

383 1993) and here we demonstrate how one may get misleading findings from a study when using

384 an unsuitable test.

385 The work in this manuscript extends upon the work of Taylor and Mackinnon (2012) and

386 Koopman et al. (2015) by examining the performance of permutation methods under a broader

387 set of conditions including the presence of covariates and confounders. Our findings support

388 prior works that also demonstrated permutation approaches outperform the more commonly-

389 used bootstrap methods in terms of excess type I error rates. This study was, however, limited

390 in scope to conditions with continuous variables and normally distributed errors, and while

391 permutation methods would be expected to outperform many other methods under more

392 challenging conditions (e.g. non-normally distributed errors), this remains yet to be

393 demonstrated. Further, there is growing enthusiasm for the use of Bayesian methods as an

394 alternative to bootstrapping when testing mediation in small samples (Nuijten, Wetzels, Matzke,

395 Dolan, \& Wagenmakers, 2015; Yuan \& MacKinnon, 2009), and recent contributions in this area 
396 include an R package, BayesMed, for implementation (Nuijten, Wetzels, Matzke, Dolan, \&

397 Wagenmakers, 2015). Future work in this area should further explore both permutation and

398 Bayesian approaches under conditions with non-normal outcomes and violations to traditional 399 regression modeling assumptions.

400

\section{Conclusions}

402

Permutation testing has been proposed as a solution to small sample mediation testing

403 (Koopman et al., 2015). Here, we evaluate two novel permutation approaches for testing the

404 indirect effect in mediation analysis (IERM and PSRM) and compare test performance to the

405 standard method (IEFM). The PSRM maintains nominal type I error rates under more

406 conditions than the IEFM or bootstrap methods, and does not substantially decrease power,

407 even in small samples. We recommend the proposed PSRM approach over the existing IEFM

408 approach for mediation analysis in studies with small sample sizes. 


\section{Acknowledgements}

410 The authors thank Brent Pedersen for use of his R code to perform Freedman and Lane

411 permutation approach available at github.com/brentp/rstuff/blob/master/perm p.R 


\section{References}

413 Anderson, M. J., \& Legendre, P. (1999). An empirical comparison of permutation methods for

414 tests of partial regression coefficients in a linear model. Journal of Statistical

$415 \quad$ Computation and Simulation, 62(3), 271-303.

416 https://doi.org/10.1080/00949659908811936

417 Aroian, L. (1944). The probability function of the product of two normally distributed variables. 418 Annals of Mathematical Statistics, 18, 265-271.

419 Brader, T., Valentino, N. A., \& Suhay, E. (2008). What Triggers Public Opposition to 420 Immigration? Anxiety, Group Cues, and Immigration Threat. American Journal of Political Science, 52(4), 959-978. https://doi.org/10.1111/j.1540-5907.2008.00353.x

422 423

424 425

426

427

428

429

430

431

432

433

Churchill, G. A., \& Doerge, R. W. (2008). Naive Application of Permutation Testing Leads to Inflated Type I Error Rates. Genetics, 178(1), 609-610. https://doi.org/10.1534/genetics.107.074609

Freedman, D., \& Lane, D. (1983). A Nonstochastic Interpretation of Reported Significance Levels. Journal of Business \& Economic Statistics, 1(4), 292. https://doi.org/10.2307/1391660

Hayes, A. F. (2009). Beyond Baron and Kenny: Statistical Mediation Analysis in the New Millennium. Communication Monographs, 76(4), 408-420. https://doi.org/10.1080/03637750903310360

Koopman, J., Howe, M., Hollenbeck, J. R., \& Sin, H.-P. (2015). Small sample mediation testing: Misplaced confidence in bootstrapped confidence intervals. Journal of Applied Psychology, 100(1), 194-202. https://doi.org/10.1037/a0036635 
434 Mackinnon, D. P., \& Fairchild, A. J. (2009). Current Directions in Mediation Analysis. Current 435 Directions in Psychological Science, 18(1), 16. https://doi.org/10.1111/j.1467-

436 8721.2009.01598.x

437

438

439

440

441

442

443

444

445

446

447

448

449

450

451

452

453

454

455

456

MacKinnon, D. P., Lockwood, C. M., Hoffman, J. M., West, S. G., \& Sheets, V. (2002). A comparison of methods to test mediation and other intervening variable effects. Psychological Methods, 7(1), 83-104.

MacKinnon, D. P., Lockwood, C. M., \& Williams, J. (2004). Confidence Limits for the Indirect Effect: Distribution of the Product and Resampling Methods. Multivariate Behavioral Research, 39(1), 99-128. https://doi.org/10.1207/s15327906mbr3901_4

Manly, B. F. (1997). Randomization and Monte Carlo methods in biology (2nd ed.). New York, NY: Chapman \& Hall.

Nuijten, M. B., Wetzels, R., Matzke, D., Dolan, C. V., \& Wagenmakers, E.-J. (2015). A default Bayesian hypothesis test for mediation. Behavior Research Methods, 47(1), 85-97. https://doi.org/10.3758/s13428-014-0470-2

Nuijten, M. B., Wetzels, R., Matzke, D., Dolan, C. V., \& Wagenmakers, E. J. (2015). BayesMed: Default Bayesian Hypothesis Tests for Correlation, Partial Correlation, and Mediation. R package version 1.0.1. https://CRAN.R-project.org/package=BayesMed

Taylor, A. B., \& MacKinnon, D. P. (2012). Four applications of permutation methods to testing a single-mediator model. Behavior Research Methods, 44(3), 806-844. https://doi.org/10.3758/s13428-011-0181-x

ter Braak, C. J. F. (1992). Permutation versus bootstrap significance tests in multiple regression and ANOVA. In Bootstrapping and related techniques (pp. 79-86). Berlin: SpringerVerlag. 
457 Tofighi, D., \& MacKinnon, D. P. (2011). RMediation: An R package for mediation analysis $458 \quad$ confidence intervals. Behavior Research Methods, 43(3), 692-700. 459 https://doi.org/10.3758/s13428-011-0076-x

460 VanderWeele, T, V., S. (2009). Conceptual issues concerning mediation, interventions and $461 \quad$ composition. Statistics and Its Interface, 2, 457-468.

462 Wagner, B. D., Kroehl, M., Gan, R., Mikulich-Gilbertson, S. K., Sagel, S. D., Riggs, P. D., ... 463 Zerbe, G. O. (2017). A Multivariate Generalized Linear Model Approach to Mediation $464 \quad$ Analysis and Application of Confidence Ellipses. Statistics in Biosciences.

$465 \quad$ https://doi.org/10.1007/s12561-017-9191-2

466 Westfall, P. H., \& Young, S. S. (1993). Resampling-based multiple testing: Examples and 467 methods for P-value adjustment. New York: Wiley.

468 Williams, J., \& MacKinnon, D. P. (2008). Resampling and Distribution of the Product Methods 469 for Testing Indirect Effects in Complex Models. Structural Equation Modeling: A $470 \quad$ Multidisciplinary Journal, 15(1), 23-51. https://doi.org/10.1080/10705510701758166

471 Yuan, Y., \& MacKinnon, D. P. (2009). Bayesian mediation analysis. Psychological Methods, $472 \quad$ 14(4), 301-322. https://doi.org/10.1037/a0016972

473 


\section{Table $\mathbf{1}$ (on next page)}

Table 1: Type I Error Rates for $\mathrm{C}$ as a confounder between the X-Y relationship. 
1 Table 1: Type I Error Rates for $\mathrm{C}$ as a confounder between the $\mathrm{X}-\mathrm{Y}$ relationship.

\begin{tabular}{|c|c|c|c|c|c|c|c|c|c|c|c|}
\hline & \multicolumn{8}{|c|}{ Conditions } & \multicolumn{3}{|c|}{ Type I error rates } \\
\hline & $\rho_{X C}$ & $\rho_{X M}$ & $\rho_{C M}$ & $\rho_{X Y}$ & $\rho_{C Y}$ & $\rho_{M Y}$ & $\alpha_{1}$ & $\gamma_{3}$ & IERM & PSRM & IEFM \\
\hline \multicolumn{12}{|c|}{$n=30$} \\
\hline \multicolumn{12}{|c|}{ Weak Confounder } \\
\hline \multicolumn{12}{|c|}{$\alpha_{1}=0, \gamma_{3}=0$} \\
\hline 1. & 0.15 & 0 & 0 & 0.15 & 0.15 & 0 & 0 & 0 & 0.044 & 0.005 & 0.006 \\
\hline \multicolumn{12}{|c|}{$\alpha_{1}=0, \gamma_{3} \neq 0$} \\
\hline 2. & 0.15 & 0 & 0 & 0.15 & 0.15 & 0.15 & 0 & 0.15 & 0.084 & 0.006 & 0.007 \\
\hline 3. & 0.15 & 0 & 0 & 0.15 & 0.15 & 0.3 & 0 & 0.3 & 0.196 & 0.016 & 0.016 \\
\hline 4. & 0.15 & 0 & 0 & 0.15 & 0.15 & 0.6 & 0 & 0.6 & 0.499 & 0.059 & 0.075 \\
\hline \multicolumn{12}{|c|}{ Strong Confounder } \\
\hline \multicolumn{12}{|c|}{$\alpha_{1}=0, \gamma_{3}=0$} \\
\hline 5. & 0.6 & 0 & 0 & 0.6 & 0.6 & 0 & 0 & 0 & 0.070 & 0.006 & 0.008 \\
\hline \multicolumn{12}{|c|}{$\alpha_{1}=0, \gamma_{3} \neq 0$} \\
\hline 6. & 0.6 & 0 & 0 & 0.6 & 0.6 & 0.15 & 0 & 0.15 & 0.127 & 0.010 & 0.011 \\
\hline 7. & 0.6 & 0 & 0 & 0.6 & 0.6 & 0.3 & 0 & 0.3 & 0.300 & 0.028 & 0.035 \\
\hline 8. & 0.6 & 0 & 0 & 0.6 & 0.6 & 0.6 & 0 & 0.6 & 0.653 & 0.068 & 0.090 \\
\hline \multicolumn{12}{|c|}{$n=100$} \\
\hline \multicolumn{12}{|c|}{ Weak Confounder } \\
\hline \multicolumn{12}{|c|}{$\alpha_{1}=0, \gamma_{3}=0$} \\
\hline 9. & 0.15 & 0 & 0 & 0.15 & 0.15 & 0 & 0 & 0 & 0.081 & 0.002 & 0.002 \\
\hline \multicolumn{12}{|c|}{$\alpha_{1}=0, \gamma_{3} \neq 0$} \\
\hline 10. & 0.15 & 0 & 0 & 0.15 & 0.15 & 0.15 & 0 & 0.15 & 0.177 & 0.014 & 0.010 \\
\hline 11. & 0.15 & 0 & 0 & 0.15 & 0.15 & 0.3 & 0 & 0.3 & 0.452 & 0.044 & 0.043 \\
\hline 12. & 0.15 & 0 & 0 & 0.15 & 0.15 & 0.6 & 0 & 0.6 & 0.719 & 0.063 & 0.068 \\
\hline \multicolumn{12}{|c|}{ Strong Confounder } \\
\hline \multicolumn{12}{|c|}{$\alpha_{1}=0, \gamma_{3}=0$} \\
\hline 13. & 0.6 & 0 & 0 & 0.6 & 0.6 & 0 & 0 & 0 & 0.049 & 0.003 & 0.003 \\
\hline \multicolumn{12}{|c|}{$\alpha_{1}=0, \gamma_{3} \neq 0$} \\
\hline 14. & 0.6 & 0 & 0 & 0.6 & 0.6 & 0.15 & 0 & 0.15 & 0.255 & 0.026 & 0.021 \\
\hline 15. & 0.6 & 0 & 0 & 0.6 & 0.6 & 0.3 & 0 & 0.3 & 0.583 & 0.047 & 0.048 \\
\hline 16. & 0.6 & 0 & 0 & 0.6 & 0.6 & 0.6 & 0 & 0.6 & 0.783 & 0.051 & 0.056 \\
\hline
\end{tabular}

2

3 * Under 1000 simulations with a binary outcome of accept or reject, deviations in Type I error 4 rates beyond a Wald confidence interval $(0.036,0.064)$ suggest deviations from a level 0.05 5 test not due to sampling. Scenarios where the error rates exceeded the Wald confidence 6 bounds are bolded. 
Table 2 (on next page)

Table 2: Power for $\mathrm{C}$ as a confounder between the $\mathrm{X}-\mathrm{Y}$ relationship 
1 Table 2: Power for $\mathrm{C}$ as a confounder between the $\mathrm{X}-\mathrm{Y}$ relationship

\begin{tabular}{|c|c|c|c|c|c|c|c|c|c|}
\hline \multicolumn{8}{|c|}{ Conditions } & \multicolumn{2}{|c|}{ Power } \\
\hline$\rho_{X C}$ & $\rho_{X M}$ & $\rho_{C M}$ & $\rho_{X Y}$ & $\rho_{C Y}$ & $\rho_{M Y}$ & $\alpha_{1}$ & $\gamma_{3}$ & PSRM & IEFM \\
\hline \multicolumn{10}{|c|}{$n=30$} \\
\hline \multicolumn{10}{|c|}{ Weak Confounder } \\
\hline 0.15 & 0.15 & 0 & 0.15 & 0.15 & 0.15 & 0.1535 & 0.1335 & 0.014 & 0.019 \\
\hline 0.15 & 0.15 & 0 & 0.15 & 0.15 & 0.3 & 0.1535 & 0.287 & 0.056 & 0.062 \\
\hline 0.15 & 0.15 & 0 & 0.15 & 0.15 & 0.6 & 0.1535 & 0.5941 & 0.119 & 0.149 \\
\hline 0.15 & 0.3 & 0 & 0.15 & 0.15 & 0.15 & 0.3069 & 0.1221 & 0.043 & 0.048 \\
\hline 0.15 & 0.3 & 0 & 0.15 & 0.15 & 0.3 & 0.3069 & 0.2873 & 0.125 & 0.134 \\
\hline 0.15 & 0.3 & 0 & 0.15 & 0.15 & 0.6 & 0.3069 & 0.6177 & 0.363 & 0.413 \\
\hline 0.15 & 0.6 & 0 & 0.15 & 0.15 & 0.15 & 0.6138 & 0.1136 & 0.074 & 0.104 \\
\hline 0.15 & 0.6 & 0 & 0.15 & 0.15 & 0.3 & 0.6138 & 0.351 & 0.313 & 0.354 \\
\hline 0.15 & 0.6 & 0 & 0.15 & 0.15 & 0.6 & 0.6138 & 0.8259 & 0.931 & 0.951 \\
\hline \multicolumn{10}{|c|}{ Strong Confounder } \\
\hline 0.6 & 0.15 & 0 & 0.6 & 0.6 & 0.15 & 0.2344 & 0.0972 & 0.016 & 0.020 \\
\hline 0.6 & 0.15 & 0 & 0.6 & 0.6 & 0.3 & 0.2344 & 0.2526 & 0.064 & 0.073 \\
\hline 0.6 & 0.15 & 0 & 0.6 & 0.6 & 0.6 & 0.2344 & 0.5636 & 0.193 & 0.232 \\
\hline 0.6 & 0.3 & 0 & 0.6 & 0.6 & 0.15 & 0.4688 & 0.0436 & 0.045 & 0.047 \\
\hline 0.6 & 0.3 & 0 & 0.6 & 0.6 & 0.3 & 0.4688 & 0.2182 & 0.175 & 0.196 \\
\hline 0.6 & 0.3 & 0 & 0.6 & 0.6 & 0.6 & 0.4688 & 0.5673 & 0.553 & 0.607 \\
\hline 0.6 & 0.6 & 0 & 0.6 & 0.6 & 0.15 & 0.9375 & -0.171 & 0.127 & 0.153 \\
\hline 0.6 & 0.6 & 0 & 0.6 & 0.6 & 0.3 & 0.9375 & 0.1714 & 0.146 & 0.181 \\
\hline 0.6 & 0.6 & 0 & 0.6 & 0.6 & 0.6 & 0.9375 & 0.8571 & 1.000 & 1.000 \\
\hline \multicolumn{10}{|c|}{$n=100$} \\
\hline \multicolumn{10}{|c|}{ Weak Confounder } \\
\hline 0.15 & 0.15 & 0 & 0.15 & 0.15 & 0.15 & 0.1535 & 0.1335 & 0.112 & 0.103 \\
\hline 0.15 & 0.15 & 0 & 0.15 & 0.15 & 0.3 & 0.1535 & 0.287 & 0.263 & 0.259 \\
\hline 0.15 & 0.15 & 0 & 0.15 & 0.15 & 0.6 & 0.1535 & 0.5941 & 0.327 & 0.340 \\
\hline 0.15 & 0.3 & 0 & 0.15 & 0.15 & 0.15 & 0.3069 & 0.1221 & 0.159 & 0.151 \\
\hline 0.15 & 0.3 & 0 & 0.15 & 0.15 & 0.3 & 0.3069 & 0.2873 & 0.715 & 0.707 \\
\hline 0.15 & 0.3 & 0 & 0.15 & 0.15 & 0.6 & 0.3069 & 0.6177 & 0.888 & 0.892 \\
\hline 0.15 & 0.6 & 0 & 0.15 & 0.15 & 0.15 & 0.6138 & 0.1136 & 0.146 & 0.160 \\
\hline 0.15 & 0.6 & 0 & 0.15 & 0.15 & 0.3 & 0.6138 & 0.351 & 0.794 & 0.806 \\
\hline 0.15 & 0.6 & 0 & 0.15 & 0.15 & 0.6 & 0.6138 & 0.8259 & 1.000 & 1.000 \\
\hline \multicolumn{10}{|c|}{ Strong Confounder } \\
\hline 0.6 & 0.15 & 0 & 0.6 & 0.6 & 0.15 & 0.2344 & 0.0972 & 0.126 & 0.105 \\
\hline 0.6 & 0.15 & 0 & 0.6 & 0.6 & 0.3 & 0.2344 & 0.2526 & 0.439 & 0.439 \\
\hline 0.6 & 0.15 & 0 & 0.6 & 0.6 & 0.6 & 0.2344 & 0.5636 & 0.476 & 0.490 \\
\hline 0.6 & 0.3 & 0 & 0.6 & 0.6 & 0.15 & 0.4688 & 0.0436 & 0.099 & 0.099 \\
\hline 0.6 & 0.3 & 0 & 0.6 & 0.6 & 0.3 & 0.4688 & 0.2182 & 0.758 & 0.762 \\
\hline
\end{tabular}




\begin{tabular}{|rrrrrrrr|rr|}
0.6 & 0.3 & 0 & 0.6 & 0.6 & 0.6 & 0.4688 & 0.5673 & 0.970 & 0.974 \\
\hline 0.6 & 0.6 & 0 & 0.6 & 0.6 & 0.15 & 0.9375 & -0.171 & 0.323 & 0.339 \\
0.6 & 0.6 & 0 & 0.6 & 0.6 & 0.3 & 0.9375 & 0.1714 & 0.314 & 0.326 \\
0.6 & 0.6 & 0 & 0.6 & 0.6 & 0.6 & 0.9375 & 0.8571 & 1.000 & 1.000 \\
\hline
\end{tabular}

2

3

4 
Table 3 (on next page)

Table 3: Type I Error Rates for Bootstrap Simulations. 
1 Table 3: Type I Error Rates for Bootstrap Simulations.

2

\begin{tabular}{|c|c|c|c|c|c|c|c|c|c|c|c|c|}
\hline & \multicolumn{7}{|c|}{ Conditions } & \multicolumn{5}{|c|}{ Type I error rates } \\
\hline$\rho_{X C}$ & $\rho_{X M}$ & $\rho_{C M}$ & $\rho_{X Y}$ & $\rho_{C Y}$ & $\rho_{M Y}$ & $\alpha_{1}$ & $\gamma_{3}$ & PSRM & IEFM & PB & BCB & BCAB \\
\hline \multicolumn{13}{|c|}{$n=30$} \\
\hline 0.15 & 0 & 0 & 0.15 & 0.15 & 0.6 & 0 & 0.6 & 0.071 & 0.086 & 0.065 & 0.115 & 0.109 \\
\hline 0.6 & 0 & 0 & 0.6 & 0.6 & 0.6 & 0 & 0.6 & 061 & 0.079 & 0.064 & 100 & 0.084 \\
\hline \multicolumn{13}{|c|}{$\mathrm{n}=100$} \\
\hline 0.15 & 0 & 0 & 0.15 & 0.15 & 0.6 & 0 & 0.6 & 0.052 & 0.056 & 0.060 & 0.090 & 0.075 \\
\hline 0.6 & 0 & 0 & 0.6 & 0.6 & 0.6 & 0 & 0.6 & 0.051 & 0.059 & 0.060 & 0.082 & 0.065 \\
\hline
\end{tabular}

3

* Under 1000 simulations with a binary outcome of accept or reject, deviations in Type I error

5 rates beyond a Wald confidence interval $(0.036,0.064)$ suggest deviations from a level 0.05

6 test not due to sampling. Scenarios where the error rates exceeded the Wald confidence

7 bounds are bolded. 
Table 4 (on next page)

Table 4: Power for Bootstrap Simulations 
1 Table 4: Power for Bootstrap Simulations

2

\begin{tabular}{|c|c|c|c|c|c|c|c|c|c|c|c|c|}
\hline & \multicolumn{7}{|c|}{ Conditions } & \multicolumn{5}{|c|}{ Power } \\
\hline$\rho_{X C}$ & $\rho_{X M}$ & $\rho_{C M}$ & $\rho_{X Y}$ & $\rho_{C Y}$ & $\rho_{M Y}$ & $\alpha_{1}$ & $\gamma_{3}$ & PSRM & IEFM & PB & BCB & BCAB \\
\hline \multicolumn{13}{|c|}{$n=30$} \\
\hline 0.15 & 0.3 & 0 & 0.15 & 0.15 & 0.6 & 0.3069 & 0.6177 & 0.335 & 0.387 & 0.339 & 0.601 & 0.423 \\
\hline 0.6 & 0.3 & 0 & 0.6 & 0.6 & 0.6 & 0.4688 & 0.5673 & 0.540 & 0.586 & 0.532 & 0.771 & 0.602 \\
\hline 0.15 & 0.6 & 0 & 0.15 & 0.15 & 0.6 & 0.6138 & 0.8259 & 0.942 & 0.953 & 0.931 & 0.982 & 0.952 \\
\hline \multicolumn{13}{|c|}{$n=100$} \\
\hline 0.15 & 0.3 & 0 & 0.15 & 0.15 & 0.3 & 0.3069 & 0.2873 & 0.682 & 0.680 & 0.660 & 0.883 & 0.760 \\
\hline 0.6 & 0.3 & 0 & 0.6 & 0.6 & 0.3 & 0.4688 & 0.2182 & 0.774 & 0.773 & 0.773 & 0.923 & 0.827 \\
\hline 0.15 & 0.6 & 0 & 0.15 & 0.15 & 0.3 & 0.6138 & 0.3510 & 0.812 & 0.825 & 0.812 & 0.917 & 0.826 \\
\hline
\end{tabular}

3 


\section{Table 5 (on next page)}

Table 5: Results from Framing of Media Stories Analysis 
1 Table 5: Results from Framing of Media Stories Analysis

2

\begin{tabular}{|lcccc|}
\hline Education Level & $\mathrm{n}$ & $\begin{array}{c}\text { IE } \\
\text { Estimate }\end{array}$ & $\begin{array}{c}\text { PSRM } \\
\text { p-value }\end{array}$ & $\begin{array}{c}\text { IEFM } \\
95 \% \mathrm{Cl}\end{array}$ \\
\hline Less than High School & 20 & 0.343 & 0.2617 & $(-0.225,1.06)$ \\
High School & 92 & 0.138 & 0.0616 & $(-0.006,0.321)$ \\
Some College & 70 & 0.439 & 0.0030 & $(0.158,0.776)$ \\
Bachelor's (or higher) & 83 & 0.190 & 0.1855 & $(-0.080,0.501)$ \\
\hline
\end{tabular}

3 
Figure 1

Figure 1: Direct and indirect effects of $X$ on $Y$ through $M$.

(A) The total effect of exposure $X$ on outcome $Y$. (B) The indirect effect of the exposure $X$ on the outcome $Y$ through the mediator $M$ is represented by the path $X->M-Y$ and the direct effect of the exposure $X$ on the outcome $Y$ not through the mediator $M$ is represented by the path $\mathrm{X}-\mathrm{Y}$.

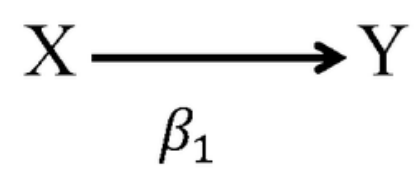

A. Total Effect

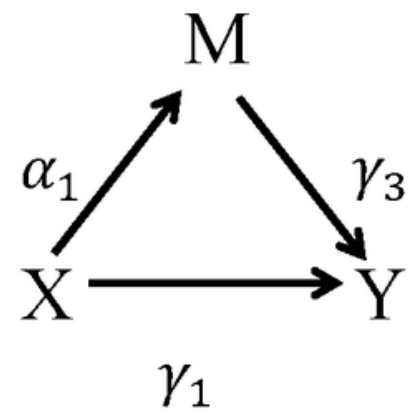

B. Direct and Indirect Effects 
Figure 2

Figure 2: Relationships between exposure, mediator, outcome, and covariate.

(A) C as a covariate, (B) C as a confounder of the $\mathrm{X}$-Y relationship, (C) C as a confounder of the M-Y relationship.
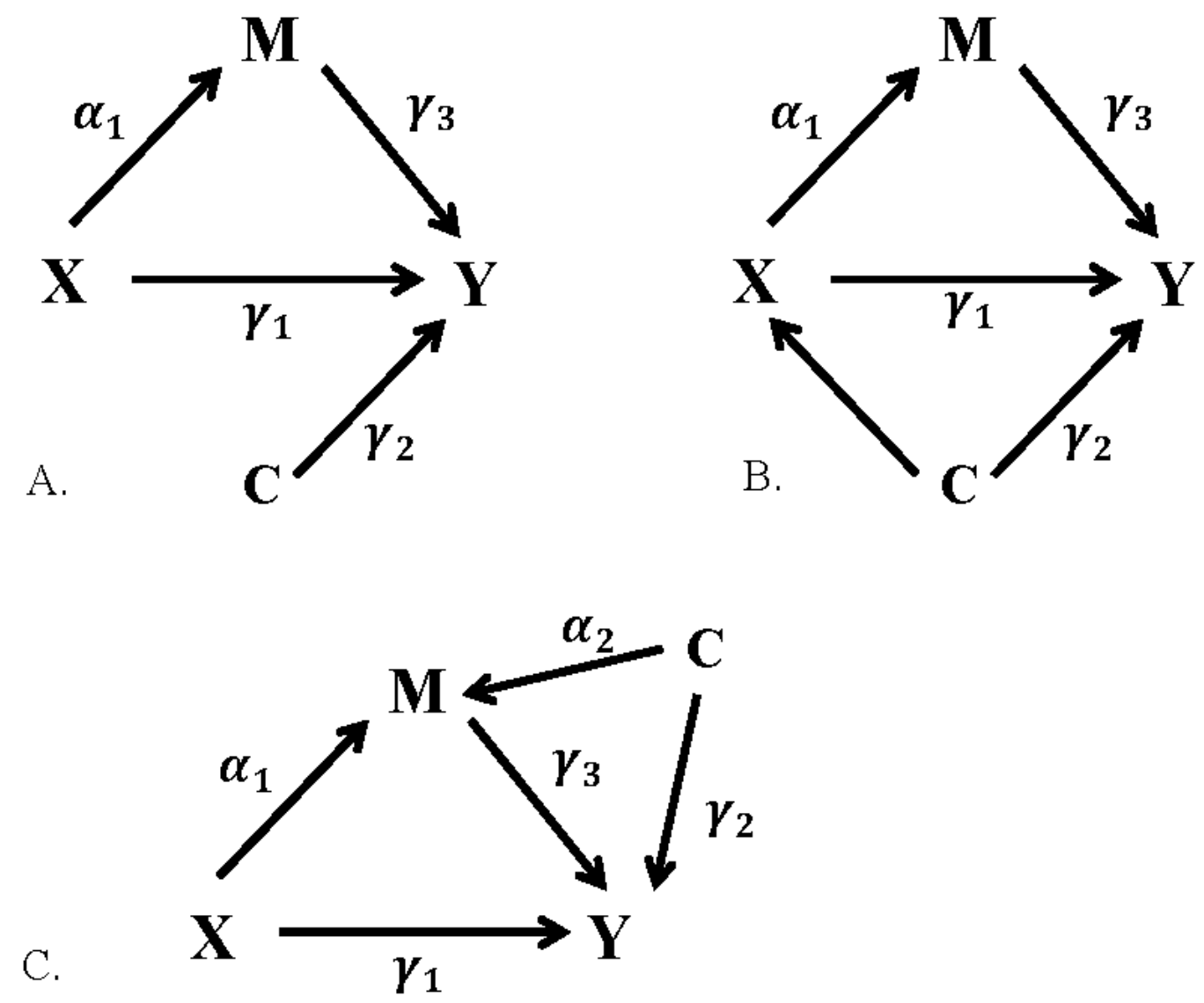
Figure 3

Figure 3. Power for test of mediation given the scenario where $\mathrm{C}$ as a weak $\mathrm{X}-\mathrm{Y}$ Confounder.

The IEFM approach is represented by the blue line and the proposed PSRM approach is represented by the green line. As seen in the plots below, the power is comparable for these 2 approaches for sample sizes of $(A) n=30$ and $(B) n=100$.
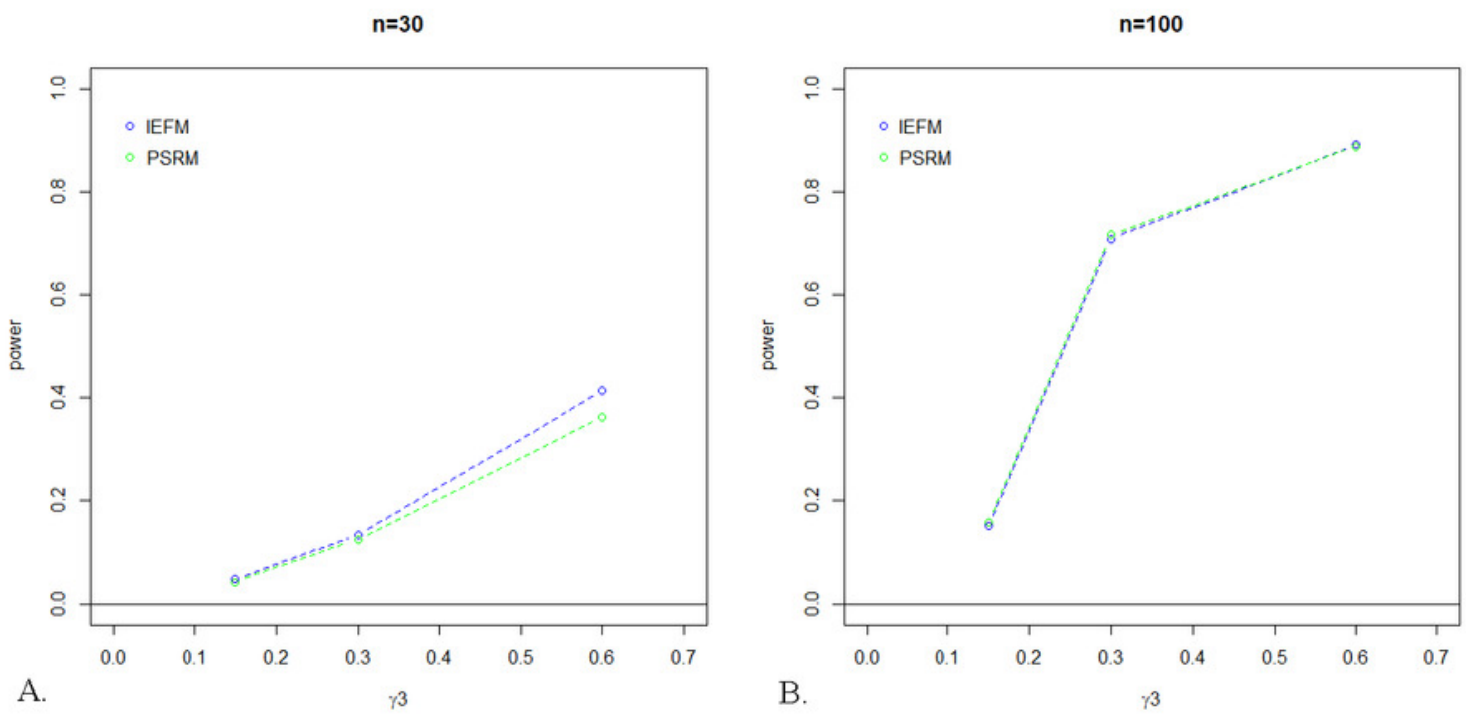


\section{Figure 4}

Figure 4. Bubble plot displaying the increase in power for PSRM as strength of coefficients increase.

The different patterns correspond to differences in the strength of the confounder and magnitude of the correlation between $\mathrm{X}$ and $\mathrm{M}$, and bubble size represents power. The bubble in the lowest left corner corresponds to the first row of results for $n=100$ in Table 2, representing $\mathrm{C}$ as a weak confounder with correlations of $\mathrm{X}-\mathrm{M}$ and $\mathrm{M}-\mathrm{Y}$ simulated to be 0.15 , and a power of $11.2 \%$. Note that, because data were generated based on correlation structures, the simulated coefficient values were sometimes different for a weak versus strong confounder. 


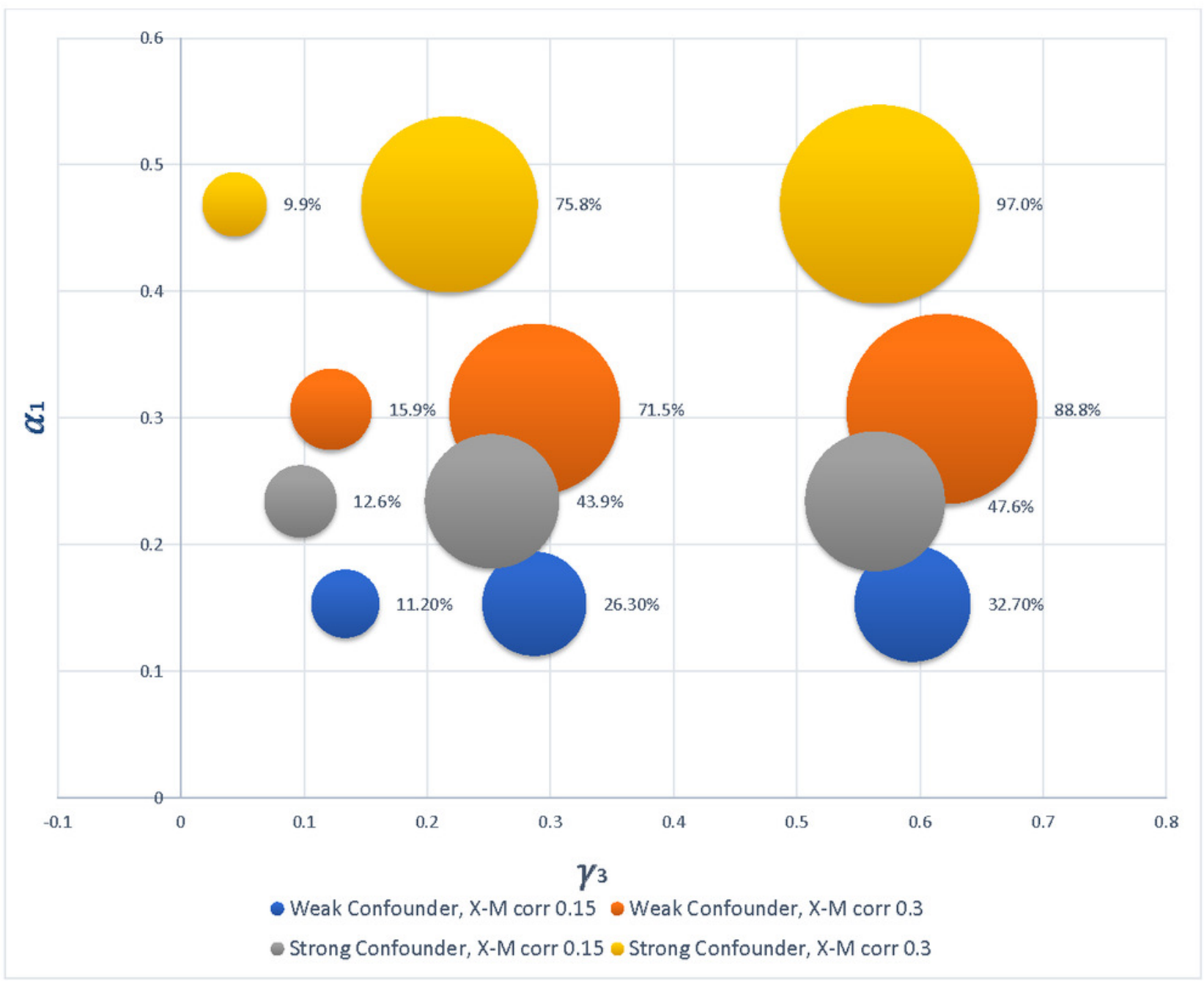

